\title{
Variation in the Caudal Branches of the External Carotid Artery: Comparison of Sex and Side
}

\author{
Authors: Sara Heltzel ${ }^{1}$, Leandra Jelinek ${ }^{2}$, David Jaynes ${ }^{3}$ \\ ${ }^{1}$ Center for Applied Biomechanics, University of Virginia, Charlottesville, Virginia, 22904 \\ email: sbh8m@virginia.edu
}

${ }^{2}$ The Edward Via College of Osteopathic Medicine, Carolinas Campus, 350 Howard Street, Spartanburg, SC, 29303

email: ljelinek@carolinas.vcom.edu

${ }^{3}$ Department of Biomedical Sciences, The Edward Via College of Osteopathic Medicine, Carolinas Campus, 350 Howard Street, Spartanburg, SC, 29303

email: djaynes@carolinas.vcom.edu

phone: (864) 327-9826

Corresponding Author: David Jaynes ${ }^{3}$

Short Title - External carotid artery branch variation

\begin{abstract}
-
Background: The branching pattern of vessels emerging from the external carotid artery varies considerably. Knowledge of this variation is critical for clinicians who perform neck procedures. This study assessed the origin of the four primary caudal branches of the external carotid artery (superior thyroid, lingual, facial, and occipital arteries) with regard to sex and side. The position of common carotid artery bifurcation was also analyzed with regard to sex and side.

Methods: The carotid arterial system of 79 cadavers from the eastern United States (37 male, 42 female) was dissected bilaterally. The position of carotid bifurcation relative to a midpoint between the suprasternal notch and mastoid process was measured with digital fractional calipers. Origin of the caudal branches was determined and the distance from the carotid bifurcation measured.

Results: On the right, the superior thyroid artery emerged from the carotid bifurcation/external carotid more frequently than it did from the common carotid (67\% and 31\%, respectively); however, on the left, it emerged more frequently from the common carotid (57\% and $43 \%$, respectively) $(p=0.003)$. No side-dependent differences were observed for the remaining three caudal branches. The occipital artery emerged below the origin of the facial artery more frequently than at/above (55\% and $44 \%$, respectively). No significant differences were found for the origin of the caudal four branches with regard to sex. There was no significant difference in the location of the carotid bifurcation with regard to sex or side.

Conclusions: The origin of the superior thyroid artery differed when comparing neck side, while the origin of the occipital artery frequently emerged caudal to that of the facial artery. Both findings are inconsistent with most anatomical atlases. The positions of the carotid bifurcation as well as the origin of the remaining caudal branches were no different with regard to either parameter.
\end{abstract}

Keywords-external carotid artery; arterial variation; sex; side; superior thyroid artery; occipital artery 


\section{Introduction}

The carotid arterial system is the main arterial blood supply to the head and neck. The common carotid artery (CCA) bifurcates to form the internal (ICA) and external (ECA) carotid arteries at roughly the level of the third or fourth cervical vertebra. The ECA typically presents eight vessels (six branches and two terminal arteries formed by its bifurcation near the temporomandibular joint). Each branch of the ECA supplies a specific region of the neck, face, and/or head; however, numerous anastomoses exist both ipsilaterally and contralaterally.

Previous studies have shown that multiple ECA branching patterns exist (Gupta et al. 2014, Hayashi et al. 2005, Kamenskiy et al. 2012, Kitagawa 1993, Klosek and Rungruang 2008, Lo et al 2006, LuČev et al. 2000, Natsis et al. 2011, Ozgur, Govsa, and Ozgur 2008, Sanjeev et al. 2010, Shintani et al. 1999, Toni et al. 2004, Vázquez et al.2009, Yonenaga et al. 2011, Zümre et al. 2005). Classic textbook representations of the ECA and the origins of its eight branches vary significantly depending on source (Drake, Vogl, and Mitchell 2005, Gilroy et al. 2008, Moore, Dalley, and Agur 2009, Netter 2010). Variation in vessel origin has been reported most frequently for the superior thyroid (STA), lingual (LA), facial (FA), and occipital (OA) arteries. Some anatomical atlases present the more common variations of certain arteries (e.g., Netter's representation of the superior mesenteric artery); however, carotid arterial system variation is not commonly depicted. Furthermore, while a few alternate patterns may be shown in some atlases, the high number of possibilities precludes their inclusion. In some cases, these variations are surprisingly common.

The STA is most commonly shown to emerge from the ECA, although it is occasionally shown arising at the carotid bifurcation (CB) (Drake, Vogl, and Mitchell 2005, Gilroy et al. 2008, Moore, Dalley, and Agur 2009, Netter 2010). The LA and FA typically originate as individual ECA branches superior to the origin of the STA, although they often originate from a common trunk (lingual-facial trunk) (Hayashi et al. 2005, Lo et al. 2006, LuČev et al. 2000, Ozgur, Govsa, and Ozgur 2008, Sanjeev et al. 2010, Shintani et al. 1999, Zümre et al. 2005). The lingual-facial trunk is omitted in most anatomy texts. The OA is the most caudal posterior branch and is commonly depicted as originating in close proximity to the origin of the FA; however, studies have shown the OA to have a variety of origin locations ranging from at/above the facial artery origin to well below the origin of the lingual artery (Hayashi et al. 2005, Shintani et al. 1999).

Standard anatomical and surgical landmarks may be ineffective or misleading in deducing vessel identity, placement, and course. The inability to anticipate the precise location of the origin and course of blood vessels make surgical procedures potentially dangerous; thus, arteries exhibiting unpredictable patterns increase the likelihood of iatrogenic injury (Chleborad and Dawson 1990, Malnar et al. 2010, Mangla and Sclafani 2008). Additionally, while arterial variation often goes unrecognized throughout an individual's life, pathological processes such as vascular disease render these variations important with regard to effective clinical intervention (Gluncic 2001, Kamenskiy et al. 2012). Emergency surgical intervention is often necessary in instances of neck trauma. Injuries involving the carotid arterial system often present as a complex cause of exsanguinating hemorrhage (Mangla and Sclafani 2008), and involvement of branches of the ECA are more common than involvement of the ECA itself (Nicoucar et al. 2008). Massive hemorrhage from carotid arterial system laceration makes surgical intervention immediately necessary to achieve hemodynamic stability; unfortunately, the likelihood of iatrogenic injury increases with further surgical intervention (Mangla and Sclafani 2008).

While variability regarding arterial characteristics (e.g., wall thickness, vessel diameter) between populations (sex, ethnicity) has been directly examined in some arterial systems (Eden et al. 2008, Lindekleiv et al. 2010, Müller et al. 1991, Schulz and Rothwell 2001), very little has been reported contrasting the differences in ECA branching pattern between sex and side. This study assessed the origin of the caudal four ECA branches to compare the anatomy of males versus females, and right versus left neck. We found that the STA origin location and distance from the carotid bifurcation (CB) varied significantly between sides (but not between sexes). To our knowledge, this is the first report of quantified asymmetry in the ECA branching pattern. In contrast to that presented in popular anatomy texts, the OA frequently originated below the origin of the FA. No significant difference was found for the origins or distance from the CB for the LA, FA or OA with regard to sex or side. Additionally, the $\mathrm{CB}$ did not differ in position with regard to sex or side.

\section{Methods}

\subsection{Subjects}

Dissection of the carotid arterial system was performed bilaterally on 79 embalmed human cadavers ( 37 male; 42 female) at four Virginia universities to quantify ECA branching pattern with regard to sex and side (71 right; 68 left). The specimens were predominantly Caucasian (77 Caucasian, 1 African American, and 1 Asian American). Digital images were taken with a Nikon Coolpix S560 camera (Nikon, Japan).

\subsection{Design}

The location of $\mathrm{CB}$ was determined in relation to the midpoint located between the mastoid process of the temporal bone and the suprasternal notch (Figure 1). This approach is typical of that used by vascular. To account for any discrepancy in neck position (due to stiffness of cadavers), the length from the mastoid process to the suprasternal notch was measured on each side and the average distance was determined. This average distance was then halved to determine the midpoint; position of the $\mathrm{CB}$ was measured from the midpoint. 
Figure 1. Determination of the midpoint between the mastoid process of the temporal bone and the suprasternal notch. Vascular surgeons approximate the location of the CB between the suprasternal notch and the mastoid process. The length of an oblique line from the mastoid process to the suprasternal notch was measured on each side. The average was determined and this distance halved to determine the midpoint (star) for each specimen.

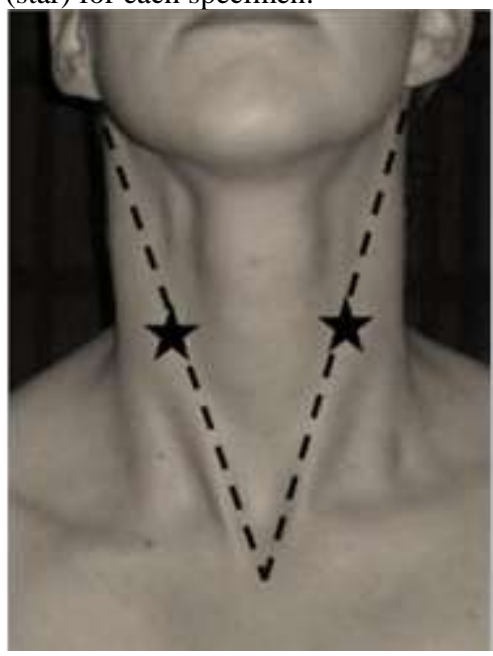

The distance between the CB (i.e., top of fork) and the origins of the STA, LA, FA and OA were measured in millimeters using electronic General No. 147 Digital Fractional Calipers (New York, USA). If the LA and FA shared a common origin, the distance between the $\mathrm{CB}$ and the origin of the common lingual-facial trunk was recorded.

The origins of the caudal four branches were categorized as follows (Figure 2):

- The origin of the STA (arising from the CB/ECA; from the CCA).

- The origin of the LA and FA (individual origins; arising from a common trunk).

- The origin of the OA in relation to that of the FA origin (level of/above; below).

Figure 2. Diagrams of the variation in origin of the caudal four branches of the external carotid artery. (A) Variation in the origin of the STA: 1) from the ECA, 2) from the level of the CB, and 3) from the CCA. STAs originating from the ECA or CB were combined in this study. (B) and (C) Variation in the LA and FA origins. (B) LA and FA originating individually from the ECA. (C) LA and FA originating as a common lingual-facial trunk. (D) Variation in the origin of the OA relative to the origin of the FA: a) above, b) at the level of, and c) below. OAs originating at or above the level of FA origin were combined in this study (bracket). $\mathrm{CCA}=$ common carotid artery; $\mathrm{CB}=$ carotid bifurcation; ECA = external carotid artery; STA = superior thyroid artery; LA = lingual artery; FA = facial artery; OA = occipital artery.
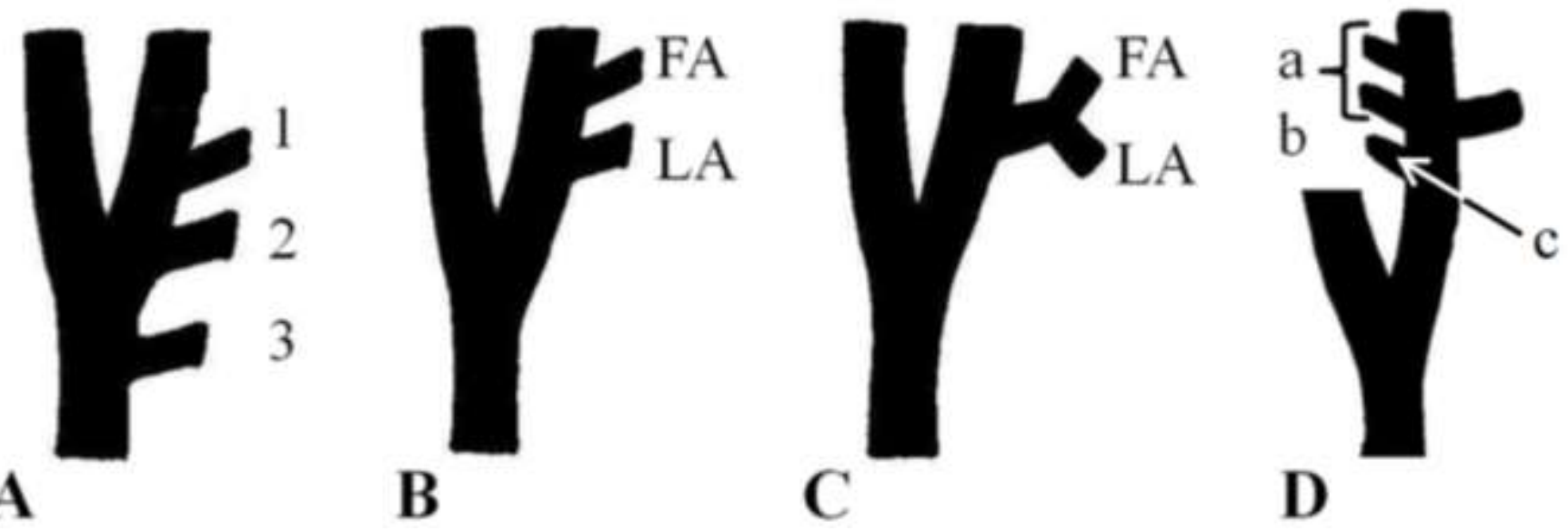


\subsection{Statistical Analyses}

Statistical analyses were performed using PASW version 18.0 statistical analysis software. Means, standard deviations, and ranges were determined for all measurements, as well as for each sex and side. The continuous variables (i.e., the position of $\mathrm{CB}$, and the distances between the CB and the STA, LA, FA and OA) were assessed by conducting repeated measures ANOVA analyses. The categorical variables (i.e., the origin of the STA, LA, FA and OA) were assessed by conducting Fisher exact tests (Kirkman, 1996). Differences were considered statistically significant at the $95 \%$ confidence interval.

\section{Results}

All CB were observed cranial to the midpoint between the mastoid process and the suprasternal notch (Table 1). No significant difference was found for the mean distance from the midpoint to the $\mathrm{CB}$ with regard to $\operatorname{sex}(\mathrm{p}=0.70)$ or side $(\mathrm{p}=$ 0.75). The STA on the right side emerged from the CB/ECA more frequently than it did from the CCA ( $67 \%$ and $31 \%$, respectively); however, on the left side, the STA emerged more frequently from the CCA than from the CB/ECA ( $57 \%$ and $43 \%$, respectively; $p=$ 0.003) (Figure 3A-3B). The cranial-caudal range of STA emergence relative to the $\mathrm{CB}$ on the right side was $8 \mathrm{~mm}$ above to $14 \mathrm{~mm}$ below; on the left side, the range was $9 \mathrm{~mm}$ above to 21 $\mathrm{mm}$ below (Figure 4). These side-dependent differences were statistically significant $(\mathrm{p}=0.006)$ (Tables $2-3)$.

Table 1. Location of the carotid bifurcation in relation to the midpoint located between the mastoid process and the suprasternal notch. Males exhibited a significantly greater mastoid process to suprasternal notch distance ( $\sim 8 \%$ longer $)$ and a significantly higher midpoint $(\sim 8 \%$ higher $)$ than females. No sex/side interaction was found for any distances, therefore, data was combined and compared separately based on sex and side. Distance in millimeters. $\mathrm{MP}=$ mastoid process; Sup. Notch = suprasternal notch; $\mathrm{CB}=$ common carotid bifurcation.

\begin{tabular}{|c|c|c|c|}
\hline & MP to Sup. Notch & Midpoint & Midpoint to CB \\
\hline & Mean +/- SD & Mean +/- SD & Mean +/-SD \\
\hline Total $(n=112-114)$ & $171.97+/-16.05$ & $85.99+/-7.96$ & $24.66+/-8.50$ \\
\hline Males $(n=25-27)$ & $* 179.62+/-16.63$ & $* 89.81+/-8.20$ & $24.12+/-7.92$ \\
\hline Females $(\mathrm{n}=25-28)$ & $* 165.46+/-12.50$ & $* 82.73+/-6.22$ & $24.94+/-9.06$ \\
\hline Right $(\mathrm{n}=58-59)$ & $171.95+/-16.10$ & $86.02+/-7.99$ & $24.40+/-9.06$ \\
\hline Left $(n=54-55)$ & $173.13+/-16.12$ & $85.95+/-8.00$ & $24.66+/-7.94$ \\
\hline
\end{tabular}

${ }^{*} p<0.05$ 
Figure 3. Photographs showing the origins of the caudal four branches of the ECA. (A) and (B) are from the same female cadaver; insets are schematic drawings showing LA and FA branching patterns. (A) Female, right neck; 1) STA emerging from ECA and a common LA/FA trunk (*denotes LA/FA trunk). (B) Female, left neck; shows STA emerging from CCA and 2) individual LA and FA origins from the ECA. (C) and (D) are from the same male cadaver; insets are schematic drawings showing typical and atypical STA, LA, and FA branching patterns. (C) Male, right neck; shows OA emerging well below FA. (D) Male, left neck; shows STA and LA originating from a common thyrolingual trunk branching from the CCA (*denotes thyrolingual trunk). CCA = common carotid artery; ECA = external carotid artery; STA = superior thyroid artery; LA= lingual artery; $\mathrm{FA}=$ facial artery; $\mathrm{OA}=$ occipital artery.
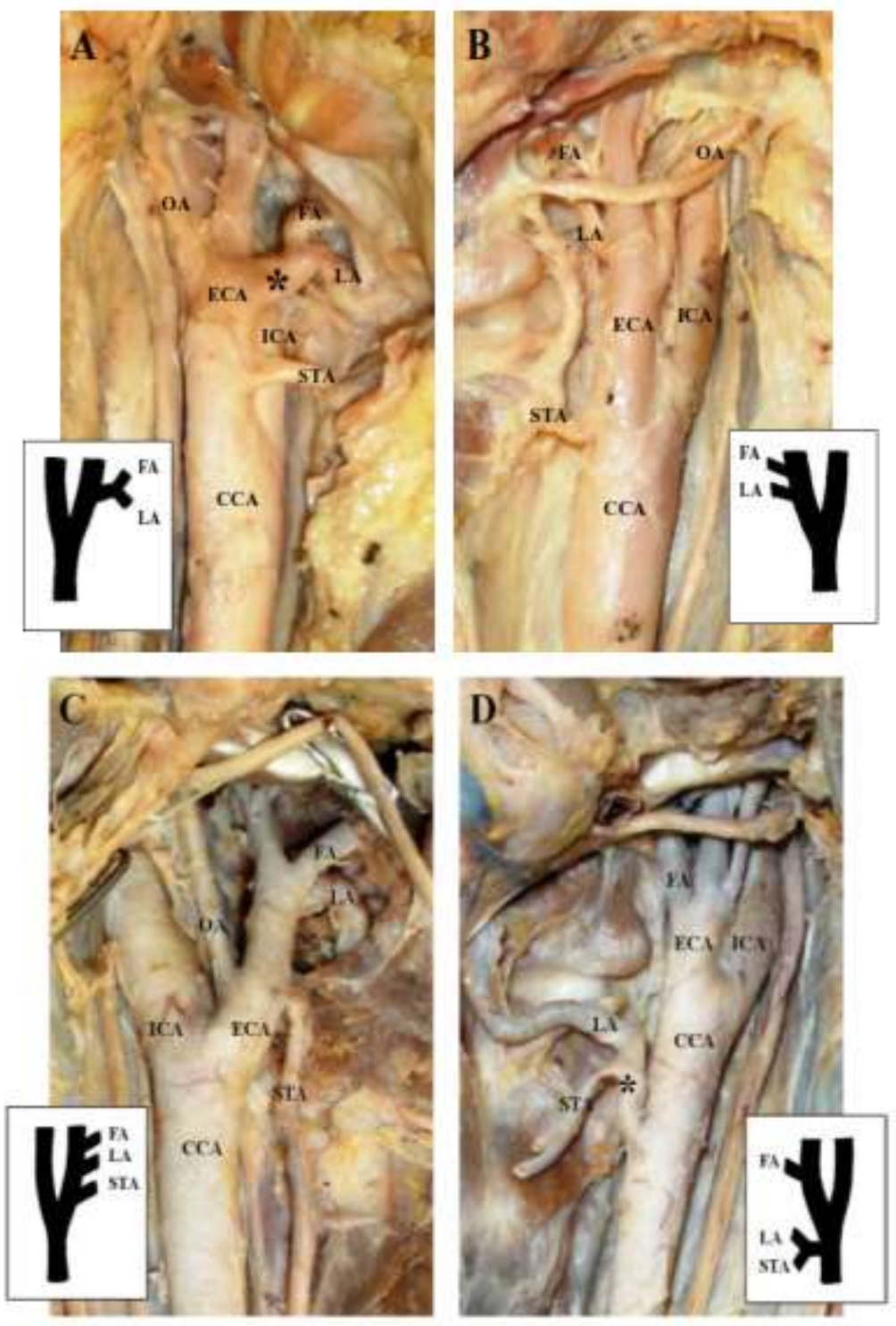
Figure 4. Diagram illustrating the range of superior thyroid artery origin. (A) Right side; STA origin location ranged from $8 \mathrm{~mm}$ cranial to CB (origin from ECA) to $14 \mathrm{~mm}$ caudal to $\mathrm{CB}$ (origin from CCA). (B) Left side; STA origin location ranged from $9 \mathrm{~mm}$ cranial to $\mathrm{CB}$ (origin from $\mathrm{ECA}$ ) to $21 \mathrm{~mm}$ caudal to $\mathrm{CB}$ (origin from $\mathrm{CCA}$ ). $\mathrm{CCA}=$ common carotid artery; $\mathrm{CB}=$ carotid bifurcation; ECA = external carotid artery; $\mathrm{STA}=$ superior thyroid artery.

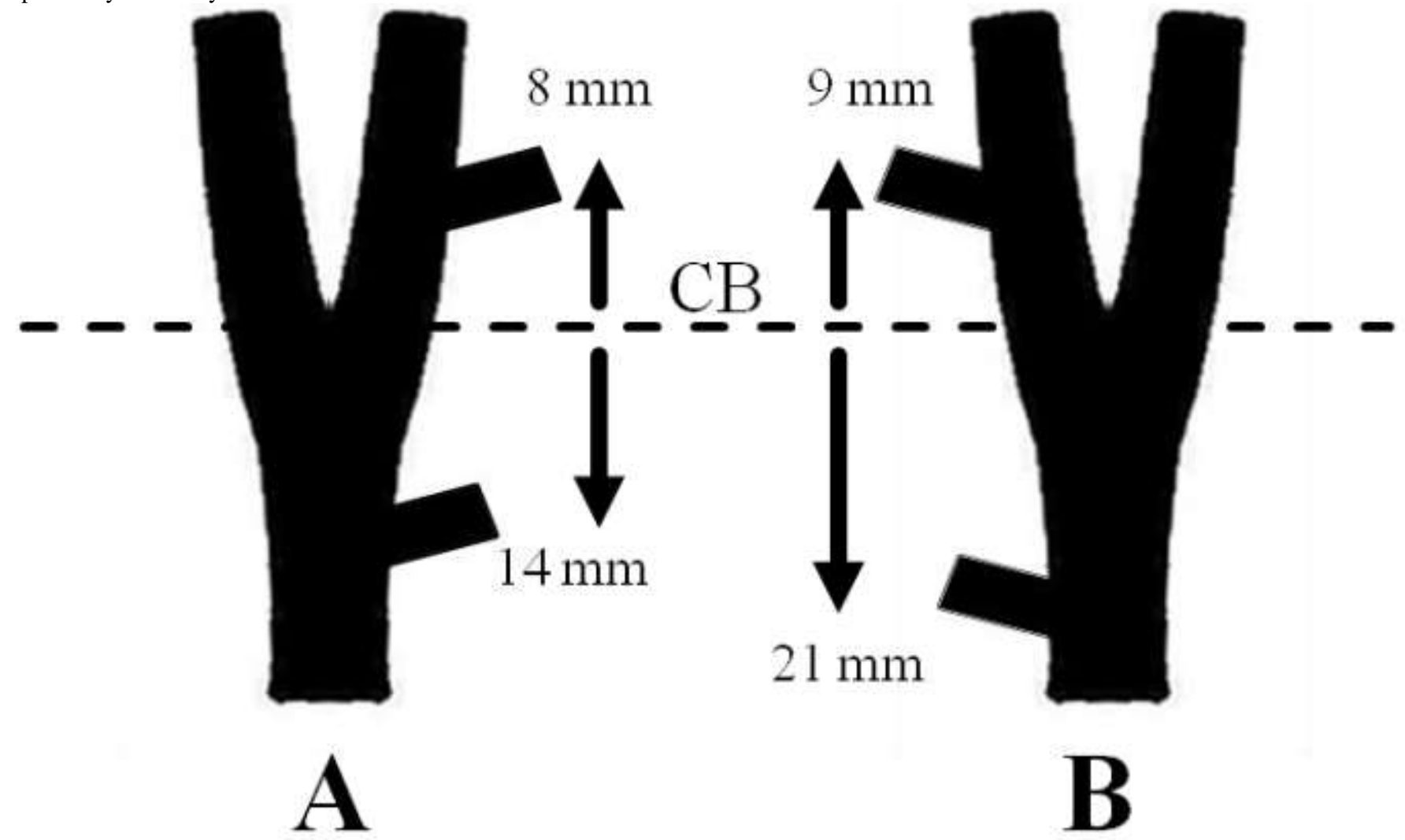

Table 2. Distances of the four major caudal branches of the external carotid artery from the common carotid bifurcation. Significant differences between right and left side were noted for the CB to STA distance. STA = superior thyroid artery; LA = lingual artery; FA = facial artery; OA = occipital artery. Distance in millimeters.

\begin{tabular}{|c|c|c|c|c|}
\hline & STA & LA & FA & $\mathrm{OA}$ \\
\hline & Mean $+/$-SD & Mean +/-SD & Mean +/-SD & Mean +/-SD \\
\hline Total $(\mathrm{n}=124-126)$ & $3.87+/-3.77$ & $12.21+/-6.49$ & $17.64+/-8.21$ & $16.68+/-8.80$ \\
\hline Males $(n=25-27)$ & $4.09+/-4.18$ & $12.73+/-6.61$ & $18.09+/-8.59$ & $17.28+/-9.49$ \\
\hline Females $(n=25-28)$ & $3.40+/-3.33$ & $12.28+/-6.42$ & $18.16+/-7.87$ & $15.65+/-8.18$ \\
\hline Right $(n=58-59)$ & $* 2.77+/-2.63$ & $12.53+/-6.46$ & $18.57+/-7.57$ & $17.13+/-8.79$ \\
\hline Left $(n=54-55)$ & $* 4.73+/-4.37$ & $12.47+/-6.56$ & $17.68+/-8.86$ & $15.80+/-8.79$ \\
\hline
\end{tabular}

${ }^{*} p<0.05$ 
Table 3. Comparison of origin of ECA caudal branches between sexes and neck side. No significant difference in origin was observed with regard to sex for the STA, LA, FA or OA. Significant difference was found for the STA origin location with regard to side. STA = superior thyroid artery; LA = lingual artery; $\mathrm{FA}=$ facial artery; $\mathrm{OA}=$ occipital artery.

\begin{tabular}{|c|c|c|c|c|c|}
\hline & TOTAL & MALE & FEMALE & RIGHT & LEFT \\
\hline STA & $(n=138)$ & $(n=66)$ & $(n=72)$ & $(n=70)$ & $(n=68)$ \\
\hline $\mathrm{CB} / \mathrm{ECA}$ & 76 & 35 & 41 & $* 47$ & $* 29$ \\
\hline $\mathrm{CCA}$ & 61 & 31 & 30 & 22 & 39 \\
\hline Other & 1 & 0 & 1 & 1 & 0 \\
\hline LA & $(n=139)$ & $(n=60)$ & $(n=73)$ & $(n=71)$ & $(n=68)$ \\
\hline Individual & 107 & 49 & 58 & 51 & 56 \\
\hline Common Trunk & 29 & 15 & 14 & 18 & 11 \\
\hline Other & 3 & 2 & 1 & 2 & 1 \\
\hline FA & $(n=136)$ & $(n=66)$ & $(n=70)$ & $(n=69)$ & $(n=67)$ \\
\hline Individual & 107 & 51 & 56 & 51 & 56 \\
\hline Common Trunk & 29 & 15 & 14 & 18 & 11 \\
\hline $\mathrm{OA}$ & $(n=124)$ & $(n=61)$ & $(n=63)$ & $(n=64)$ & $(n=60)$ \\
\hline At/Above FA & 55 & 28 & 27 & 31 & 24 \\
\hline Below FA & 68 & 32 & 36 & 33 & 35 \\
\hline Other & 1 & 1 & 0 & 0 & 1 \\
\hline
\end{tabular}

${ }^{*} p<0.05$

For the remaining parameters, no statistically significant differences were found for sex or side; therefore, the data were combined before analysis. The LA and FA arose individually more frequently than from a common trunk $(79 \%$ and $21 \%$, respectively). The OA emerged below the origin of the FA more frequently than at/above the FA (55\% and 44\%, respectively) (Figure 3C). Although not statistically significant, the OA frequently emerged below the FA, which contrasts with that typically presented in anatomical atlases.

Three specimens (2\%) displayed thyrolingual trunks, in which the LA and STA shared a common origin. One thyrolingual trunk originated from the ECA, one from the $\mathrm{CB}$, and one from the CCA. While a thyrolingual trunk originating from the ECA has an incidence rate of approximately $2.5 \%$ (Ozgur, Govsa, and Ozgur 2008), one originating from the CCA has been reported to occur in less than $0.1 \%$ of specimens (Lippert and Pabst 2011) (Figure 3D).

\section{Discussion}

Knowledge of arterial variations and their probabilities is essential to reduce the incidence of iatrogenic injury during surgery, particularly those performed without the opportunity to evaluate arterial patterns beforehand. Branches of the carotid arterial system are of special interest since they frequently vary and are located in a vulnerable region of the body. Variation in pattern of the caudal four vessels of the ECA has been well documented; however, to our knowledge, no study has been conducted to assess variation as a function of sex and/or side. We found no statistically significant difference in any arterial origin or position of the $\mathrm{CB}$ with regard to sex; however, the STA origin and distance from the $\mathrm{CB}$ varied significantly between sides. On the left, the STA arose most frequently from the CCA, while on the right the STA arose most frequently from the CB/ECA. These findings contrast with the commonly taught pattern that the STA arises from the ECA bilaterally. This asymmetry could be due to the 1) difference in CCA anatomy (origin, length) when comparing sides, 2) dynamics/timing of the vascularization of the two lobes of the thyroid gland during/following its' descent, 
and/or 3) some other factor (e.g., mechanisms controlling vessel formation).

Although not statistically significant, we did observe that, bilaterally, the OA frequently originated below the FA than at/above the FA. This finding differs with most illustrations in popular atlases. Our observation that the FA independently originated from the ECA more frequently than from a common trunk with the LA is consistent with reports of other authors. No significant difference was found for the origin, or the distance from the CB for the LA, FA or OA with regard side or sex.

Although a recent study proposed (for embryologic reasons) that the $\mathrm{CB}$ is part of the $\mathrm{CCA}$, and should therefore be considered as such when measurements are taken (Natsis et al. 2011), we chose to evaluate our data as we did to compare/contrast our findings with that presented in widely used atlases. Specifically, the STA is predominantly shown emerging from the ECA or $\mathrm{CB}$; therefore, to clearly distinguish the differences, we assessed the origin of the STA as arising from the $\mathrm{CB} / \mathrm{ECA}$ or from the CCA.
We conclude that variation in ECA branching pattern differs in some aspects with regard to side (e.g., STA), but not with sex. Our findings that the 1) STA originates more frequently from the CCA on the left side only and 2) the OA frequently emerges caudal to the facial artery (bilaterally) is in disagreement with the majority of representations shown in commonly used human anatomy atlases. It is possible these discrepancies exist due to the populations studied (Gupta et al. 2014, Ongeti and Ogeng'o 2012). Recognition of the likelihood of these variations is an important step in reducing morbidity associated with surgical and radiological procedures of the neck.

\section{Acknowledgements}

The authors would like to thank Dr. Robert Lee of James Madison University for assistance with the statistics. The authors would also like to thank the Edward Via College of Osteopathic Medicine - Virginia Campus, Eastern Virginia Medical School, Virginia Commonwealth University, and James Madison University for access to cadavers. 


\section{References}

Chleborad, W. P., and Dawson, D. L. 1990. The profunda femoris artery: variations and clinical applications. Clin Anat, 3:33-40.

Drake, R. L, Vogl, W., and Mitchell, A. W. 2005. Gray's anatomy for students. 1st Ed. Philadelphia: Churchill Livingstone, pp. $909-912$.

Eden, S. V., Meurer, W. J., Sánchez, B. N., Lisabeth L. D., Smith, M. A., Brown, D. L., and Morgenstern, L. B. 2008. Gender and ethnic differences in subarachnoid hemorrhage. Neurology, 71:731-735.

Gilroy, A., MacPherson, B., Ross, L., Schuenke, M., Schulte, E., and Schumacher, U. (2008) Atlas of anatomy. 1st Ed. New York: Thieme Medical Publishers, pp. 491-495.

Gluncic, V., Petanjek, Z., Marusic, A., and Gluncic, I. 2001. High bifurcation of common carotid artery, anomalous origin of ascending pharyngeal artery and anomalous branching pattern of external carotid artery. Surg Radiol Anat, 23:123-125.

Gupta, P., Bhalla, A. S., Thulkar, S., Kumar, A., Mohanti, B. K., Thakar, A., and Sharma, A. 2014. Variations in superior thyroid artery: A selective angiographic study. Indian J Radiol Imaging, 24:66-71.

Hayashi, N., Hori, E., Ohtani, Y., Ohtani, O., Kuwayama, N., and Endo, S. 2005. Surgical anatomy of the cervical carotid artery for carotid endarterectomy. Neurol Med Chir (Tokyo), 45:25-9; discussion 30.

Kamenskiy, A. V., MacTaggart, J. N., Pipinos, I. I., Bikhchandani, J., and Dzenis, Y. A. 2012. Three-dimensional geometry of the human carotid artery. J Biomech Eng 134, (6), 064502.

Kirkman, T. W. 1996. Statistics to use. URL: http://www.physics.csbsju.edu/stats/.

Kitagawa, W. 1993. Arterial supply of the thyroid gland in the human fetuses. Nippon Ika Daigaku Zasshi, 60:140-155.

Klosek, S. K., and Rungruang, T. 2008. Topography of carotid bifurcation: considerations for neck examination. Surg Radiol Anat, 30:383-387.

Lindekleiv, H. M., Valen-Sendstad, K., Morgan, M. K., Mardal, K., Faulder, K., Magnus, J. H., Waterloo, K., Romner, B., and Ingebrigtsen, T. 2010. Sex differences in intracranial arterial bifurcations. Gend Med, 7:149-155.

Lippert, H., and Pabst, R. 2011. Arterial Variations in Man: Classification and Frequency. 1st Ed. J.F. Bergmann-Verlag.

Lo, A., Oehley, M., Bartlett, A., Adams, D., Blyth, P., and Al-Ali, S. 2006. Anatomical variations of the common carotid artery bifurcation. ANZ J Surg, 76: 970-972.

LuČev, N., Bobinac, D., Marič, I., and Drešćik, I. 2000. Variations of the great arteries in the carotid triangle. Otolaryngol Head Neck Surg, 122:590-591.

Malnar, D., Klasan, G. S., Miletić, D., Bajek, S., Vranić, T. S., Arbanas, J., Bobinac, D., and Čoklo, M. 2010. Properties of the celiac trunk anatomical study. Coll Antropol, 349:17-921.

Mangla, S., and Sclafani, S. J. A. 2008. External carotid arterial injury. Injury, 39:1249-1256.

Moore, K., Dalley, A., and Agur A. 2009. Clinically oriented anatomy. 6th Ed. Baltimore: Lippincott Williams \& Wilkins, pp. $1001-1004$.

Müller, H. R., Brunhölzl, C., Radü, E. W., and Buser, M. 1991. Sex and side differences of cerebral arterial caliber. Neuroradiology, $33: 212-216$.

Natsis, K., Raikos, A., Foundos, I., Noussios, G., Lazaridis, N., and Njau, S. N. 2011. Superior thyroid artery origin in Caucasian Greeks: A new classification proposal and review of the literature. Clin Anat, 24:699-705.

Netter, F. H. 2010. Atlas of human anatomy. 5th Ed. Teterboro: Saunders, pp. 69-71. Nicoucar, K., Popova, N., Becker, M., Dulguerov, and P. 2008. Pseudoaneurysm of the external carotid artery after a blunt facial trauma. J Trauma, 65:E24-27.

Ongeti, K. W., and Ogeng'o, J. A. 2012. Variant origin of the superior thyroid artery in a Kenyan population. Clin Anat, 25(2):198-202.

Ozgur, Z., Govsa, F., and Ozgur, T. 2008. Assessment of origin characteristics of the front branches of the external carotid artery. J Craniofac Surg, 19:1159-1166. 
Sanjeev, I. K, Anita, H., Ashwini, M., Mahesh, U., and Rairam, G. B. 2010. Branching pattern of external carotid artery in human cadavers. J Clin Diag Res, 4:3128-3133.

Schulz, U. G., and Rothwell, P. M. 2001. Sex differences in carotid bifurcation anatomy and the distribution of atherosclerotic plaque. Stroke, 32:1525-1531.

Shintani, S., Terakado, N., Alcalde, R. E., Tomizawa, K., Nakayama, S., Ueyama, Y., Ichikawa, H., Sugimoto, T., and Matsumura, T. 1999. An anatomical study of the arteries for intraarterial chemotherapy of head and neck cancer. Int J Clin Oncol, 4:327-330.

Toni, R., Della Casa, C., Castorina, S., Malaguti, A., Mosca, S., Roti, E., and Valenti, G. 2004. A meta-analysis of superior thyroid artery variations in different human groups and their clinical implications. Ann Anat, 186: 255-262.

Vázquez, T., Cobiella, R., Maranillo, E., Valderrama, F. J., McHanwell, S., Parkin, I., and Sañudo, J. R. 2009. Anatomical variations of the superior thyroid and superior laryngeal arteries. Head Neck, 31:1078-1085.

Yonenaga, K., Tohnai, I., Mitsudo, K., Mori, Y., Saijo, H., Iwai, T., Yonehara, Y., Ota, Y., Torigoe, K., Takato, T. 2011. Anatomical study of the external carotid artery and its branches for administration of superselective intra-arterial chemotherapy via the superficial temporal artery. Int J Clin Oncol, 16(6):654-659.

Zümre, Ö., Salbacak, A., Çiçekcibaşi, A., Tuncer, I., Seker, M. 2005. Investigation of the bifurcation level of the common carotid artery and variations of the branches of the external carotid artery in human fetuses. Ann of Anat, 187:361-369. 